\title{
Development of a sensitive assay for the detection of Pseudoloma neurophilia in laboratory populations of the zebrafish Danio rerio
}

\author{
Justin L. Sanders* ${ }^{*}$ Michael L. Kent \\ Department of Microbiology, Oregon State University, Corvallis, Oregon 97331, USA
}

\begin{abstract}
The zebrafish Danio rerio is an increasingly important biological model in many areas of research. Due to the potential for non-protocol-induced variation, diseases of zebrafish, especially those resulting in chronic, sub-lethal infections, are of great concern. The microsporidium Pseudoloma neurophilia is a common parasite of laboratory zebrafish. Current methods for detection of this parasite require lethal sampling of fish, which is often undesirable with poorly spawning mutant lines and small populations. We present here an improved molecular-based diagnostic assay using realtime polymerase chain reaction (PCR), and including sonication treatment prior to DNA extraction. Comparisons of several DNA extraction methods were performed to determine the method providing the maximum sensitivity. Sonication was found to be the most effective method for disrupting spores. Compared to previously published data on PCR-based assay using a dilution experiment, sensitivity is increased. This shows that our assay, which includes sonication, is capable of detecting parasite DNA at 1 log higher dilution than the conventional PCR-based assay, which does not include sonication. Furthermore, we demonstrate the application of this method to testing of water, eggs, and sperm, providing a potential non-lethal method for detection of this parasite in zebrafish colonies with a sensitivity of 10 spores $\mathrm{l}^{-1}$ of water, 2 spores per spiked egg sample, and 10 spores $\mathrm{\mu l}^{-1}$ of spiked sperm sample.
\end{abstract}

KEY WORDS: Microsporidia $\cdot$ Real-time PCR $\cdot$ Danio rerio

\section{INTRODUCTION}

The zebrafish Danio rerio is a widely used biological model in several fields, including developmental biology, immunology, toxicology, and infectious disease and cancer research (Eisen 1996, Dooley \& Zon 2000, Amatruda et al. 2002, Hill et al. 2005). Laboratory colonies of zebrafish are typically composed of specialized mutant strains of fish possessing genotypes useful to specific areas of study, and hardier wild-type strains are used for breeding stock and for maintaining specific genotypes in a heterozygotic state (Westerfield 2007). Maintenance and husbandry of many of the mutant strains is often difficult (Lawrence 2007). Individual adult fish from these lines are therefore often in limited supply and may be extremely valuable. Whereas laboratory populations of zebrafish occasion- ally are affected by acute infectious diseases, more important and more prevalent are chronic infections by Mycobacterium spp. and Pseudoloma neurophilia (Microsporidia; Matthews 2004, Kent et al. 2004, 2009). The latter has been detected in $>50 \%$ of the facilities that we have examined through the Zebrafish International Resource Center (ZIRC) diagnostic service.

Pseudoloma neurophilia, a microsporidian parasite, generally causes chronic infections in zebrafish, with clinical signs ranging from obvious scoliotic changes and emaciated appearance to subclinical infections exhibiting no outward signs (Matthews et al. 2001). As with other animals used in research, experiments utilizing zebrafish with these infections are subject to non-experimental variation, potentially confounding results, as described in laboratory colonies of rabbits and mice infected with the microsporidian parasite 
Encephalitozoon cuniculi (Baker 1998). Furthermore, fish without overt clinical disease may have reduced fecundity associated with the infection (Ramsay et al. 2009).

Microsporidia are obligate intracellular parasites with species infecting virtually all animal phyla. They have a relatively simple life cycle consisting of 2 general developmental stages, mergony and sporogony. Meronts multiply inside the infected host cell, eventually forming sporonts and then spores, which are ultimately released from the host and transmit the infection. The infectious spore stage has a thick, chitinous outer layer, making it extremely resistant to environmental stress and lysis and allowing the organism to maintain viability for extended periods in the aquatic environment (Shaw et al. 2000).

While several assays exist for the detection of pathogens in other fish species using non-lethal sampling methods (Miriam et al. 1997, López-Vázquez et al. 2006, Lindstrom et al. 2009), due to the zebrafish's small size and consequent difficulty in obtaining blood and other tissues from it, these sampling methods are generally not applicable to the zebrafish. But because zebrafish can be housed in relatively small-volume tanks, the screening of water in tanks and even effluent from flow-through systems seems a feasible method by which to detect this pathogen without requiring lethal sampling of adult fish. Moreover, zebrafish spawn frequently, and thus spawning products (sperm and egg) are readily available for testing.

For Microsporidia that infect fish, the only viable stage found in water is the spore, as other stages of these obligate intracellular parasites would not survive long outside the host. Efficient disruption of spores is therefore crucial for obtaining DNA in the development of sensitive assays that are based on detection of this stage. Disruption of the tough, chitinous spore stage of the parasite, however, requires special methods such as mechanical disruption by bead-beating or sonication (Docker et al. 1997, Dowd et al. 1998, Müller et al. 1999, Fournier et al. 2000, Graczyk et al. 2007, Hoffman et al. 2007, Phelps \& Goodwin 2007), the use of saccharolytic enzymes such as chitinase (Müller et al. 1999, Delarbre et al. 2001), or in vitro germination of the spore using chemicals such as hydrogen peroxide in combination with other purification methods (Higes et al. 2006). Additionally, the few genomic studies that have been conducted on microsporidian parasites have found a general pattern of small, streamlined genomes with very few gene copies (Williams et al. 2008). In fact, one genus has been found to possess only a single copy of the ribosomal RNA (ssu rRNA) gene (Cornman et al. 2009). Since most PCR tests for microsporidia have been based on this gene, this highlights the need to maximize spore disruption and DNA concentration to achieve a sensitive and practical method of detection (Zhu et al. 1993, Brown \& Kent 2002, Joseph et al. 2006).

Whipps \& Kent (2006) developed a PCR test for Pseudoloma neurophilia based on the detection of small subunit rDNA (ssrDNA) gene sequences, which was capable of consistently detecting down to an estimated 10 spores per sample of brain and spinal cord tissue. Here we describe a new and more sensitive approach to $P$. neurophilia, using a real-time PCR platform. Our intent was to use this real-time PCR assay for screening water and spawning products. An important focus of our study was determination of optimal extraction methods for detecting the spores in both media. We also included screening of water from spawning tanks, eggs, and sperm for non-lethal testing, as zebrafish spawn prodigiously, and spores of $P$. neurophilia occur both within and outside of eggs in ovaries (Kent \& Bishop-Stewart 2003, Kent et al. 2007).

\section{MATERIALS AND METHODS}

Pseudoloma neurophilia spore preparations. All $P$. neurophilia spores used in this study were obtained from hind brain and anterior spinal cords from 40 known infected stock zebrafish that were euthanized by an overdose of tricaine methanesulfite (Argent Laboratories). A modification of a previously described method (Ferguson et al. 2007) was used to isolate spores. Briefly, hindbrain and spinal cord tissue were mixed with $5 \mathrm{ml}$ sterile phosphate-buffered saline (PBS) and then homogenized by passing through successively smaller-gauge needles and filtered through a $20 \mu \mathrm{m}$ nylon mesh filter. This homogenate was then centrifuged through a $50 \%$ Percoll (Sigma-Aldrich) gradient for $50 \mathrm{~min}$ at $1200 \times g$ and further purified by washing in a $0.45 \mu \mathrm{m}$ filter (Millipore) twice with PBS. The purpose of this was to minimize the number of presporogonic stages present, so that results of the extraction studies would be based on spores only. The resulting spore suspension was eluted from the filter in PBS, quantified using a hemocytometer, and then diluted in PBS as needed.

Assay design. A Taqman-based PCR assay was used to measure Pseudoloma neurophilia DNA employing an ABI 7500 sequence detection system (SDS; ABI Biosystems). Primers were designed to amplify the ssuRNA gene of $P$. neurophilia based on the sequence available in the National Center for Biotechnology Information (NCBI) GenBank (accession number AF322654), and using the Primer-Basic Local Alignment Search Tool (Primer-BLAST) program, with the primer parameters set to search for a PCR product size from 70 to 125 base pairs (bp) and an optimal primer 
melting temperature of $60^{\circ} \mathrm{C}$, as well as BLAST parameters set to search for similarity in the NCBI nonredundant database for specificity (all GenBank + RefSeq Nucleotides + EMBL + DDBJ + PDB sequences, excluding HTGS0,1,2, EST, GSS, STS, PAT, WGS). The forward primer PN10F (5'-GTA ATC GCG GGC TCA CTA AG-3'), and reverse primer PN10R (5'-GCT CGC TCA GCC AAA TAA AC-3') were selected on the basis of lack of identity to related microsporidia, on annealing temperature, and on their small amplicon size of 113 bp from position 1175 to position 1288 on the ssu rRNA gene. A 3' hydrolysis probe complementary to a $23 \mathrm{bp}$ section of the amplified region was designed using the sequence 5'-6-carboxyfluorescein (FAM)-ACA CAC CGC CCG TCG TTA TCG AA-3'Black Hole Quencher 1 (BHQ1). All reactions were performed in $25 \mu \mathrm{l}$ using $900 \mathrm{nM}$ forward and reverse primers, $250 \mathrm{nM}$ of hydrolysis probe, 1× TaqMan Universal PCR Master Mix (ABI) and $2 \mu$ of sample extract. The real-time PCR was performed on an ABI 7500 SDS using the following reaction conditions: $50^{\circ} \mathrm{C}$ for $2 \mathrm{~min}, 95^{\circ} \mathrm{C}$ for $10 \mathrm{~min}$, followed by 40 repetitions at $95^{\circ} \mathrm{C}$ for $15 \mathrm{~s}$ and at $60^{\circ} \mathrm{C}$ for $1 \mathrm{~min}$. Data analysis was performed using the 7500 System SDS Software version 1.3.1 (ABI).

Cross-reactivity testing. Cross reactivity of the assay was performed using the PN10F/PN10R primer and the PN probe with DNA extracts obtained from spores of 2 fish microsporidian parasites that might be encountered in a zebrafish research facility: Glugea anomala, obtained from three-spined sticklebacks in a research colony, and Pleistophora hyphessobryconis, obtained from neon tetras from a vendor of tropical fish.

Evaluation of pretreatment and DNA extraction methods for spores. Sonication: Sonication was one of the pretreatment methods we were most interested in testing. Prior to comparing pretreatment and extraction methods in the 2 trials described in this section, it was necessary to determine the optimal sonication time for the disruption of spores. We therefore performed a time study in which suspensions of spores (consisting of 1000 spores in $100 \mu \mathrm{l}$ of PBS) were sonicated with a Branson Sonifier 250 (Branson) to various time points, and $2 \mu \mathrm{l}$ of the crude sonicate was tested by the real-time PCR method described in 'Assay design' above. The time points to which we tested were within a range of 15 to $900 \mathrm{~s}$ (see Fig. 2), all tests being run at $55 \mathrm{~W}$ and at a frequency of $20 \mathrm{kHz}$. Samples were not cooled during the sonication period, but were immediately placed on ice after treatment. The probe was decontaminated between samples using $10 \%$ household bleach followed by rinsing with sterile water. Each time point test was performed in triplicate. It was determined that 5 min consistently provided the lowest quantification cycle, and this sonication time point was therefore used in the extraction-comparison trials.

Trial A: A preliminary experiment was performed to assess the efficacy of sonication and hydrogen peroxide pretreatments in conjunction with 3 DNA extraction methods (Table 1). Trial A was performed using an initial $900 \mu \mathrm{l}$ suspension containing 30000 spores in total. The suspension was divided into three $300 \mu \mathrm{l}$ aliquots, one of which was subjected to treatment with hydrogen peroxide, another to sonication, and the third to neither.

Pretreatment with hydrogen peroxide: As numerous microsporidian spores have been shown to germinate in vitro in the presence of hydrogen peroxide (Keohane \& Weiss 1999), we attempted to germinate spores of Pseudoloma neurophilia by adding $30 \mu \mathrm{l}$ of $30 \%$ hydrogen peroxide to the spore sample for a final concentration of $9 \%$, and incubated it at $30^{\circ} \mathrm{C}$ for $30 \mathrm{~min}$. The suspension was centrifuged for $5 \mathrm{~min}$ at $14000 \times g$ in a tabletop centrifuge. The supernatant was removed, $300 \mu \mathrm{l}$ of molecular-grade water was added, and the suspension was allowed to sit for 30 min prior to further treatment.

Pretreatment with sonication: Samples were sonicated with the Branson Sonifier 250 for 5 min and im-

Table 1. Pseudoloma neurophilia. Experimental setup for 2 extraction comparison trials. Trial A samples were $300 \mu \mathrm{l}$ aliquots taken from initial pool of 30000 spores in $900 \mu \mathrm{l}$ phosphate-buffered saline (PBS). Following pretreatment, samples were divided into 6 aliquots for DNA extraction. Each extraction method was performed twice. Samples in Trial B represent individual purified spore suspensions containing 1000 spores each in $100 \mu \mathrm{l}$ PBS. Trial B procedures were performed in triplicate

\begin{tabular}{|cccc|}
\hline Trial & Sample & Pretreatment & DNA extraction \\
\hline A & 1 & None & $\begin{array}{c}\text { No extraction } \\
\text { QIAGEN } \\
\text { MoBio }\end{array}$ \\
& & & \\
& & & \\
& 2 & Hydrogen peroxide & No extraction \\
& & & QIAGEN \\
& & & MoBio \\
& & & \\
& & Sonication & No extraction \\
& & & QIAGEN \\
& & & MoBio \\
& & & \\
& & None & None \\
& & Bead-beating & QIAGEN \\
& & Chitinase & QIAGEN \\
& & None & QIAGEN \\
& & None & MoBio \\
& & Sonication & None \\
& & Sonication & QIAGEN \\
& & & \\
& & & \\
& & &
\end{tabular}


mediately placed on ice after treatment. The probe was decontaminated between samples using $10 \%$ household bleach followed by rinsing with sterile water.

Following this trial (consisting of treatment with hydrogen peroxide, treatment by sonication, and no treatment), each pool was then divided into six $50 \mu \mathrm{l}$ aliquots and either tested directly or extracted in duplicate using the following methods before testing duplicate reactions by real-time PCR:

QIAGEN: The DNeasy Blood and Tissue Extraction kit (QIAGEN) was used for this procedure, following the manufacturer's protocol for extraction of DNA from tissues, with the addition of a single overnight freezethaw of the spore suspension and an overnight Proteinase $\mathrm{K}$ and lysis buffer digestion at $56^{\circ} \mathrm{C}$. Samples were eluted in $100 \mu \mathrm{l}$ of buffer supplied in the kit.

MoBio: The UltraClean ${ }^{\mathrm{TM}}$ Microbial DNA Isolation kit (MoBio) was used for this procedure. The kit employs heat, detergent lysis, and bead-beating using specialized bead tubes and a standard vortex mixer with an adapter plate. The manufacturer's protocol was followed, using the included reagents, bead tubes, and silica membrane centrifugal filter columns. Briefly, $50 \mu \mathrm{l}$ of pretreated or non-pretreated spore suspensions were first added to detergent buffer and heated at $65^{\circ} \mathrm{C}$ for $10 \mathrm{~min}$, after which they were placed in the bead tubes and vortexed for another $10 \mathrm{~min}$. After vortexing, suspensions were added to silica membrane centrifugation filters to bind DNA and cellular components were washed out with the provided wash buffer. Finally, DNA was eluted in $50 \mu \mathrm{l}$ of Tris buffer supplied in the kit.

Trial B: Based on Trial A results, only sonication vs. no treatment was investigated further. In Trial B, sonication, accompanied now by bead-beating and the addition of chitinase, was applied to purified spore suspensions of 1000 spores in $100 \mu \mathrm{l}$ PBS. Sonication was performed as described in Trial A (Table 1). QIAGEN (with or without pretreatment) and MoBio extractions (made according to manufacturer instructions) were conducted as described in Trial A. Three spore suspensions were tested directly by real-time PCR with neither pretreatment nor DNA extraction. The following pretreatment-extraction methods were compared using spore suspensions in triplicate:

Bead-beating: Spores were suspended in Buffer Animal Tissue Lysis (ATL) with Proteinase K, both from the QIAGEN Blood and Tissue Extraction kit, and $500 \mathrm{mg}$ of $0.5 \mu \mathrm{m}$ glass beads (Sigma-Aldrich) in $1.5 \mathrm{ml}$ screw-cap tubes. The samples were then run on a bead-beater (BioSpec) at high speed (4500 oscillations $\mathrm{min}^{-1}$ ) for $3 \mathrm{~min}$, after which they were incubated for $2 \mathrm{~h}$ at $56^{\circ} \mathrm{C}$ and extracted following the QIAGEN method described above. All samples were eluted in a final volume of $100 \mu \mathrm{l}$ of buffer supplied in the QIAGEN kit.
Chitinase: Chitinase (0.4 U, Sigma-Aldrich) and $200 \mu \mathrm{l}$ potassium phosphate buffer (200 mM, pH 6.0) were added to each sample, which was then incubated at $37^{\circ} \mathrm{C}$ for $30 \mathrm{~min}$. Proteinase $\mathrm{K}$ from the DNeasy kit was then added and the samples were processed using the QIAGEN method per manufacturer's protocol, and eluted in a final volume of $100 \mu \mathrm{l}$ of buffer supplied in the QIAGEN kit.

Statistical analysis: Levene's test for equality of variances was performed on the quantification cycle threshold $\left(\mathrm{C}_{\mathrm{q}}\right)$ values obtained in Trial B. After determining that there were no significant differences between variances in the extraction methods (Levene's test, $\mathrm{p}=0.73$ ), a 1 -way analysis of variance (ANOVA) was performed. Multiple comparison with best procedure based on Hsu's method (Kuehl 2000) was performed to determine the methods which provided the highest sensitivity. All analyses were performed using the statistical package R (http://www.r-project.org).

Based on results from the extraction method study, all further experiments employed sonication only, or sonication followed by QIAGEN extraction.

Detection limit of real-time PCR. Spores in PBS: Spore suspensions at 3 concentrations were prepared in triplicate as follows: 1000 spores $100 \mathrm{\mu l}^{-1}$ PBS, 100 spores $100 \mathrm{\mu l}^{-1} \mathrm{PBS}$, and 10 spores $100 \mathrm{\mu l}^{-1}$ PBS. Each suspension was sonicated, and $2 \mu \mathrm{l}$ of the crude sonicate was directly analyzed using the real-time PCR method described in 'Assay design' above, in order to obtain 20,2 , and 0.2 spores per real-time PCR reaction, respectively.

Spores in group spawn-tank water: The capacity of the test to detect the parasite in spawn water was evaluated. Thirteen liters of fish-system water were divided into thirteen $1 \mathrm{l}$ flasks and inoculated as follows: three $1 \mathrm{l}$ aliquots with 10 spores, three $1 \mathrm{l}$ aliquots with 50 spores, three 11 aliquots with 100 spores, three $1 \mathrm{l}$ aliquots with 500 spores and one $1 \mathrm{l}$ aliquot as an uninoculated control.

Each spiked $1 \mathrm{l}$ water sample was individually filtered through a $1.2 \mu \mathrm{m}$ nitrocellulose filter (Millipore \#RAWP04700) in a fritted glass filter holder (Millipore) using a vacuum pump at $300 \mathrm{~mm} \mathrm{Hg}$. After filtration, the filter was rolled up using sterile forceps and placed in a $1.5 \mathrm{ml}$ conical screw-cap tube. The water filtration apparatus was washed and decontaminated with $10 \%$ household bleach, followed by rinsing in sterile water between samples.

One $\mathrm{ml}$ of acetone was added to each $1.5 \mathrm{ml}$ conical tube with the nitrocellulose filter and vortexed for several seconds. The tubes were then centrifuged at maximum speed for 3 min (>16000 relative centrifugal force [rcf]). The acetone supernatant was carefully aspirated off the pellet with a transfer pipette. This was performed 2 times to ensure that all dissolved nitro- 
cellulose was removed from the sample. One $\mathrm{ml}$ of $100 \%$ ethanol was then added to each tube, and the pellet was suspended by vortexing and centrifuged at $3000 \times g$ for $5 \mathrm{~min}$. The ethanol was again carefully aspirated and $5 \mathrm{ml}$ of $70 \%$ ethanol was added to each tube. Tubes were centrifuged for $5 \mathrm{~min}$ at $3000 \times g$ and the ethanol was again aspirated. The pellet was resuspended in $100 \mu \mathrm{l}$ of PBS and sonicated for $5 \mathrm{~min}$ at 55 W. After sonication, each sample was placed on ice and DNA was extracted using the QIAGEN extraction protocol described in 'QIAGEN' above. All samples were eluted in a final volume of $200 \mu$ l of buffer supplied in the kit.

Spores in eggs: We conducted the following test to evaluate detection of spores associated with eggs. A total of 2000 eggs was obtained from the specific pathogen-free (SPF) zebrafish colony at the Sinnhuber Aquatic Research Laboratory (SARL), Oregon State University, and divided into 4 aliquots of 500 eggs each, with PBS added to make a total volume of $1 \mathrm{ml}$. One aliquot was spiked with 100 spores, one with 1000 spores, one with 10000 spores, and the last aliquot was left with no spores as a negative control. Each sample was then sonicated for $5 \mathrm{~min}$ at $55 \mathrm{~W}$ and cooled on ice. After sonication, three $40 \mu$ aliquots (equaling approximately 20 eggs based on original volume, and either 4 , 40 , or 400 spores) were taken from each sample, and DNA was extracted using the QIAGEN blood and tissue protocol as described in 'QIAGEN' above. All samples were eluted in a final volume of $200 \mu$ l of buffer supplied in the kit.

Spores in sperm: Sperm was obtained by squeezing of SPF zebrafish males from the colony at the SARL. Sperm samples were pooled and divided into $5 \mu \mathrm{l}$ aliquots in $100 \mu \mathrm{l}$ of PBS and spiked, in triplicate, with 5 spores per sample, 50 spores per sample and 500 spores per sample. All samples were brought to a volume of $200 \mu \mathrm{l}$ and were then sonicated with DNA being then extracted using the QIAGEN extraction described in 'QIAGEN' above. All samples were eluted in a final volume of $200 \mu \mathrm{l}$ of buffer supplied in the kit. The spiked spawn water, eggs, and sperm were run in triplicate on different days.

Comparison with conventional PCR assay: We compared our new real-time PCR-based assay, which included pretreatment by sonication, with the previously developed conventional PCR-based test described by Whipps \& Kent (2006), which does not include sonication. The hindbrain and spinal cords from 6 infected fish were removed and individually homogenized in $100 \mu \mathrm{l}$ sterile PBS. Then $50 \mu \mathrm{l}$ of the resulting homogenate was sonicated for $5 \mathrm{~min}$, followed by purification using the QIAGEN method, and the remaining $50 \mu$ was extracted by the QIAGEN method as described by Whipps \& Kent (2006). All DNA extracts were eluted in $100 \mu$ l of Tris buffer and 4 serial 10-fold dilutions of each were made in sterile water. After extraction, all samples and dilutions were run in single reactions using real-time PCR as described here, and the conventional method as described by Whipps \& Kent (2006), with minor modification. Briefly, the primer pair PN18S5F 5'-GAA AAT TAC CGG AGC CTG AAG TC-3', and PN18S5R, 5'-TTC CCT CTC TCT CCA AAT TTC GG-3' were used to amplify a 788 bp fragment of the ssu rDNA of Pseudoloma neurophilia using conventional PCR. The reaction was carried out in $25 \mu$ l volumes using the Platinum ${ }^{\circledR}$ PCR SuperMix (Invitrogen), $12.5 \mathrm{pmol}$ of each primer, and $2 \mu \mathrm{l}$ of extracted DNA. Amplification was carried out on a PTC-200 thermocycler (MJ Research) with an initial denaturation at $94^{\circ} \mathrm{C}$ for $3 \mathrm{~min}$, followed by 35 cycles at $94^{\circ} \mathrm{C}$ for $30 \mathrm{~s}$, at $55^{\circ} \mathrm{C}$ for $30 \mathrm{~s}$, at $72^{\circ} \mathrm{C}$ for $60 \mathrm{~s}$, and a final extension step at $72^{\circ} \mathrm{C}$ for $7 \mathrm{~min}$. Products were visualized on a $1.5 \%$ agarose gel stained with $\mathrm{SYBR}^{\circledR}$ Safe DNA Gel Stain (Invitrogen). Results were reported as positive ( $P$. neurophilia ssu rDNA detected) or negative ( $P$. neurophilia ssu rDNA not detected), based on the presence or absence of a band corresponding with an approximate size of $788 \mathrm{bp}$.

Group spawn experiment: The capacity of the test to detect the parasite in spawning products of infected fish was evaluated. Ten adult zebrafish were arbitrarily selected from a population determined to be $10 \%$ infected by Pseudoloma neurophilia based on histological examination of a subsample of fish 3 mon prior to the experiment. The fish were placed in a spawning tank with $10 \mathrm{l}$ of system water overnight. The following day, the fish were collected and euthanized by an overdose of tricaine methanesulfonate. Brains and spinal tissue were collected using sterile instruments between individuals, and then were placed in separate $1.5 \mathrm{ml}$ tubes and extracted using the standard QIAGEN protocol, without sonication, with an overnight digestion at $56^{\circ} \mathrm{C}$. Water was filtered in 11 aliquots; filters were dissolved, sonicated, and extracted as described in 'Detection limits of real-time PCR. Spores in group spawntank water'. Eggs were pooled, sonicated, and five $40 \mu \mathrm{l}$ aliquots were extracted as described in 'Spores in eggs' above. All samples were tested in single reactions using the real-time PCR method.

\section{RESULTS}

Cross reactivity. Primer-BLAST search of the $20 \mathrm{bp}$ forward primer, PN10F, and the 20 bp reverse primer, PN10R, returned AF322654.1 Pseudoloma neurophilia ssu rRNA gene, partial sequence; internal transcribed spacer, complete sequence; and large subunit ribosomal lsu rRNA gene, partial sequence, with no other 
Pseudoloma neurophilia Loma embiotocia Ovipleistophora mirandellae Loma salmonae

Glugea sp.

Loma morhua

Loma psittaca

Pleistophora hyphessobryconis

Heterosporis anguillarum

Ichthyosporidium giganteum

Pleistophora mulleri

Dasyatispora levantinae

Glugea stephani

Loma salmonae

Loma sp.

Glugea anomala

Loma sp.

Glugea atherinae

Loma acerinae

Pleistophora typicalis

Pleistophora sp. (PA)

Ichthyosporidium sp.

Pseudoloma neurophilia

Loma embiotocia

Ovipleistophora mirandellae

Loma salmonae

Glugea sp.

Loma morhua

Loma psittaca

Pleistophora hyphessobryconis

Heterosporis anguillarum

Ichthyosporidium giganteum

Pleistophora mulleri

Dasyatispora levantinae

Glugea stephani

Loma salmonae

Loma sp.

Glugea anomala

Loma $\mathrm{sp}$.

Glugea atherinae

Loma acerinae

Pleistophora typicalis

Pleistophora sp. (PA)

Ichthyosporidium sp.

Pseudoloma neurophilia

Loma embiotocia

Ovipleistophora mirandellae

Loma salmonae

Glugea sp.

Loma morhua

Loma psittaca

Pleistophora hyphessobryconis

Heterosporis anguillarum

Ichthyosporidium giganteum

Pleistophora mulleri

Dasyatispora levantinae

Glugea stephani

Loma salmonae

Loma sp.

Glugea anomala

Loma sp.

Glugea atherinae

Loma acerinae

Pleistophora typicalis

Pleistophora sp. (PA)

Ichthyosporidium sp. $\ldots|\ldots| \ldots|\ldots| \ldots|\ldots| \ldots|\ldots| \ldots|\ldots| \ldots \mid$

$$
1230 \quad 1240
$$

GCTCAGGAAC GCGgAATTGT TAGTAAT-CG CGgGCTCACT AAgACGCGAT GCTCAGGAAC GAGGAATTGC TAGTAAT-CG CGGACTCATT AAGACGCGAT GCTCAGGAAC GAGGAATTGC TAGTAAT-CG CGGGCTCATT AAGACGCGAT GCTCAGGAAC GAGGAATTGC TAGTAAT-CG CGGACTCATT AAGACGCGAT GCTCAGGAAC GAGGAATTGC TAGTAAT-CG CGGACTCATT AAGACGCGAT GCTCAGGAAC GAGGAATTGC TAGTAAT-CG CGGACTCATT AAGACGCGAT GCTCAGGAAC GAGGAATTGC TAGTAAT-CG CGGGCTCATT AAGACGCGAT GCTCAGGAAC GAGGAATTGC TAGTAAT-CG CGGGCTCATT AAGACGCGAT GCTCAGGAAC GAGGAATTGC TAGTAAT-CG CGGGCTCATT AAGACGCGAT GCTCAGGAAC GTGGAATTGC TAGTAATTCG CGGACTCATT AAGACGCGAT GCTCAGGAAC GAGGAATTGC TAGTAAT-CG CGGGCTCATT AAGACGCGAT GCTCGGGAAC GAGGAATTGC TAGTAAT-CG CGGGCTCATT AAGACGCGAT GCTCAGGAAC GAGGAATTGC TAGTAAT-CG CGGACTCATT AAGACGCGAT GCTCAGGAAC GAGGAATTGC TAGTAAT-CG CGGACTCATT AAGACGCGAT GCTCAGGAAC GAGGAATTGC TAGTAAT-CG CGGACTCATT AAGACGCGAT GCTCAGGAAC GAGGAATTGC TAGTAAT-CG CGGACTCATT AAGACGCGAT GCTCAGGAAC GAGGAATTGC TAGTAAT-CG CGGACTCATT AAGACGCGAT GCTCAGGAAC GAGGAATTGC TAGTAAT-CG CGGACTCATT AAGACGCGAT GCTCGGGAAC GAGGAATTGC TAGTAAT-CG CGgGCTCATT AGGACGCGAT GCTCAGGAAC GAGGAATTGC TAGTAAT-CG CGGGCTCATT AAGACGCGAT GCTCGGGAAC GAGGAATTGC TAGTAAT-CG CGGACTCATT AAGACGCGAT GCTCAGGAAC GTGGAATTGC TAGTAAT-CG CGGACTCATT AAGACGCGAT

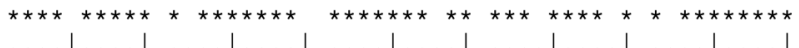
$\begin{array}{ccccc}1280 & 1290 & 1300 & 1310 & 1320\end{array}$ GAATGCGACC CTGTTCATTG T---ACACAC CGCCCGTCGT TATCGAAGAC GAATACGTCC CTGTTCTTTG T---ACACAC CGCCCGTCGT TATCGAAGAT GAATACGTCC CTGTTCTTTG T---ACACAC CGCCCGTCGT TATCGAAGAT GAATACGTCC CTGTTCTTTG T---ACACAC CGCCCGTCGT TATCGAAGAT GAATACGTCC CTGTTCTTTG T---ACACAC CGCCCGTCGT TATCGAAGAT GAATACGTCC CTGTTCTTTG T---ACACAC CGCCCGTCGT TATCGAAGAT GAATACGTCC CTGTTCTTTG T---ACACAC CGCCCGTCGT TATCGAAGAT GAATACGTCC CTGTTCTTTG T---ACACAC CGCCCGTCGT TATCGAAGAT GAATACGTCC CTGTTCTTTG T---ACACAC CGCCCGTCGT TATCGAAGAT GAATACGTCC CTGTTCTTTG T---ACACAC CGCCCGTCGT TANCGAAGAC GAATACGTCC CTGTTCTTTG T---ACACAC CGCCCGTCGT TATCGAAGAT GAATACGTCC CTGTTCTTTG T---ACACAC CGCCCGTCGT TATCGAAGAT GAATACGTCC CTGTTCTTTG T---ACACAC CGCCCGTCGT TATCGAAGAT GAATACGTCC CTGTTCTTTG T---ACACAC CGCCCGTCGT TATCGAAGAT GAATACGTCC CTGTTCTTTG T---ACACAC CGCCCGTCGT TATCGAAGAT GAATACGTCC CTGTTCTTTG TAGTACGCAC CGCCCGTCGT TATCGAAGAT GAATACGTCC CTGTTCTTTG T---ACACAC CGCCCGTCGT TATCGAAGAT GAATACGTCC CTGTTCTTTG T---ACACAC CGCCCGTCGT TATCGAAGAT GAATACGTCC CTGTTCTTTG T---ACACAC CGCCCGTCGT TATCGAAGAT GAATACGTCC CTGTTCTTTG T---ACACAC CGCCCGTCGT TATCGAAGAT GAATACGTCC CTGTTCTTTG T---ACACAC CGCCCGTCGT TATCGAAGAT GAATACGTCC CTGTTCTTTG T---ACACAC CGCCCGTCGT TATCGAATAC

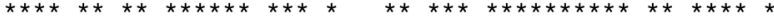

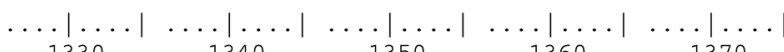
1340 1350 1360 1370

GATGCTAGGC GCG-AGCAAG GTTTATTTGG CTGAGCGAGC GCAGGGTATT GAAGACAGGC GCG-AACGAT CT---ACCA- GAAAGTGAGC GCAGGTCTTT GGAGTCAGGC GCG-AACAAG CGAGAGC--. --GAGTGAGT GCAGGATTCT GAAGATAGGC GCG-AACGAT CT---ACCA- GAAAGTGAGC GCAGGTTTTT GGAGTCAGGC GCG-AACAAG CGAGAGC--- - -GAGTGAGT GCAGGATTCT GAAGACAGGC GCG-AACGAT CT---ACCA- GAAAGTGAGC GCAGGTCTTT GGATTCAGGC GCG-AACGAG CGAGAGC-- - --GAGTGA-C GCAGGGTT-GGAGTCAGGC GCG-AACAAG CGAGAGC-.. - -GAGTAAGT GCAGGATTCT GGAGTCAGGC GCG-AACAAG CGAGAGC-- - --GAGTGAGT GCAGGGTTCT GGTGCTCGGC GCG-AGCAAG GTGAAATCA- CTGAGCGAGC GCAAGGTACC GGAGTCAGGC GCG-AACAAG CGAGAGC--- --GAGTGAGT GCAGGGTTCT GGAGTCAGGC GCG-AACAAG CGAGAGC - - - -GAGTGAGT GCAGGGCTCT GGAGTCAGGC GCG-AACAAG CGAGAGC-- - --GAGTGAGT GCAGGATTCT GAAGATAGGC GCG-AACGAT CT---ACCA- GAAAGTGAGC GCAGGTTTTT GAAGACAGGC GCG-AACGAT CTACCAGA- - - AAGTGAGC GCAGGTCTTT GGAGTCAGGC GCGGAACAAG CGAGAGC-- - - GAGTGAGT GCAGGATTCT GAAGACAGGC GCG-AACGAT CTACCAGA-- --AAGTGAGC GCAGGTCTTT GGAGTCAGGC GCG-AACAAG CGAGAGC-- - - GAGTGAGT GCAGGATTCT GGAGTCAGGC GCG-AACAAG CGAGAGC- - - -GAGTGAGT GCAGGATTCT GGAGTCAGGC GCG-AACAAG CGAGAGC-- - - -GAGTGAGT GCAGGGTTCT GGAGTCAGGC GCG-AACAAG CGAGAGC--. --GAGTAAGT GCAGGATTCT GGTGCTCGGC GCG-AGCAAG GTGAAATCA- CTGAGCGAGC GCAAGGTACC

Fig. 1. Pseudoloma neurophilia. Partial small subunit ribosomal DNA (ssu rDNA) sequence alignment of P. neurophilia (GenBank AF322654), Loma embiotocia (GenBank AF320310), Ovipleistophora mirandellae (GenBank AF356223), L. salmonae (GenBank U78736), Glugea sp. (GenBank AY090038), L. morhua (GenBank GQ121037), L. psittaca (GenBank FJ843104), Pleistophora hyphessobryconis (GenBank GU126672), Heterosporis anguillarum (GenBank AF387331), Ichthyosporidium giganteum (GenBank L13430), Pleistophora mulleri (GenBank FN434084), Dasyatispora levantinae (GenBank GU183263), Glugea stephani (GenBank AF056015), L. salmonae (GenBank HM626215), Loma sp. (GenBank HM626217), G. anomala (GenBank AF044391), Loma sp. (GenBank AF104081), G. atherinae (GenBank U15987), L. acerinae (GenBank AJ252951), Pleistophora typicalis (GenBank AJ252956), Pleistophora sp. (PA) (GenBank AJ252958), and Ichthyosporidium sp.(GenBank L39110). PN10F and PN10R primer locations are underlined and PN10 probe location is shaded. Asterisks: regions of nucleotide similarity 


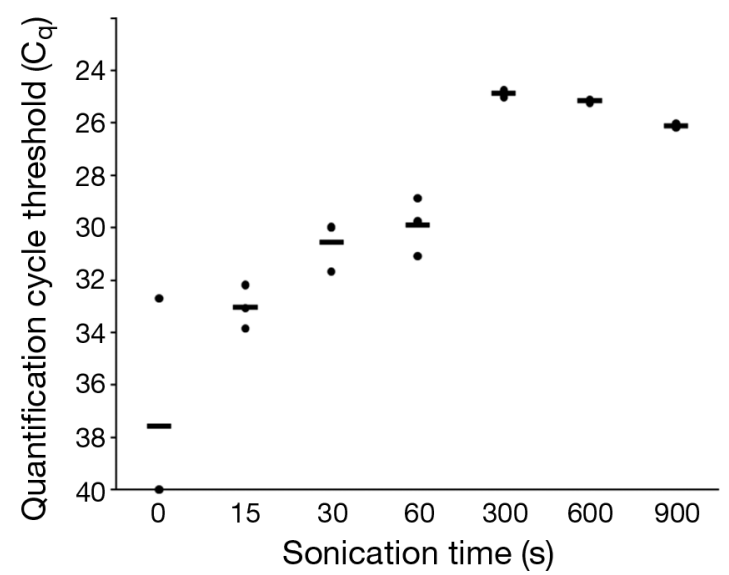

Fig. 2. Pseudoloma neurophilia. Spore sonication time study, showing quantification cycle thresholds $\left(\mathrm{C}_{\mathrm{q}}\right)$ of triplicate suspensions of 1000 spores of $P$. neurophilia sonicated at $55 \mathrm{~W}$ to different time points and tested by real-time PCR. Bars: mean $\mathrm{C}_{\mathrm{q}}$ points: individual sample $\mathrm{C}_{\mathrm{q}} \mathrm{S}$

matches found in the selected database. (All GenBank + EMBL + DDBJ + PDB sequences, but no EST, STS, GSS, environmental samples or phase 0,1 , or 2 HTGS sequences.) An alignment of partial ssu rDNA sequences of several related Microsporidia showed several nucleotide mismatches to that of $P$. neurophilia present within the $113 \mathrm{bp}$ amplicon in regions specific to both primers and probe sequences (Fig. 1). Testing of DNA extracted from spores of Glugea anomala and Pleistophora hyphessobryconis resulted in no increase in fluorescence detected through 40 cycles of real-time PCR.

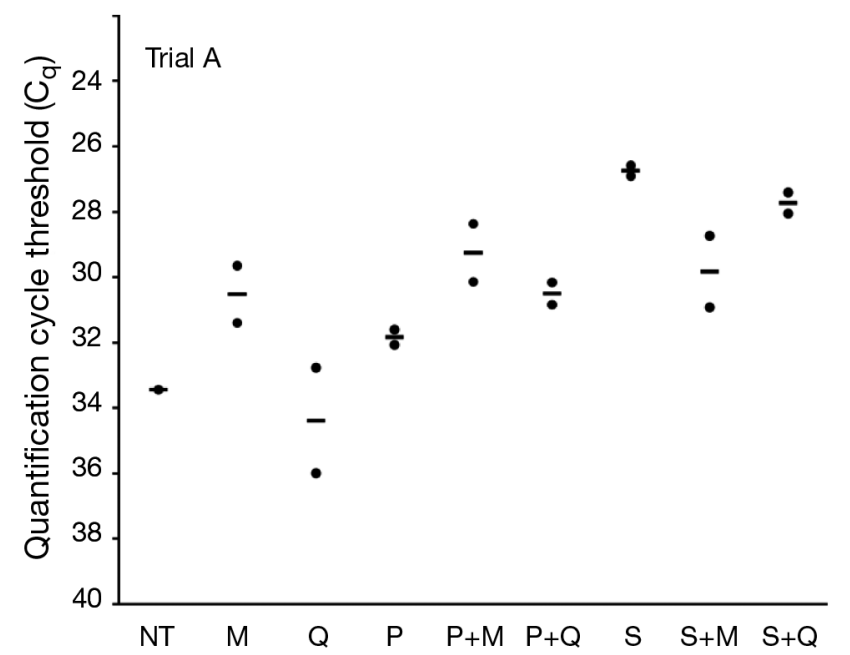

Sonication time study. Sonification for $5 \mathrm{~min}$ at $55 \mathrm{~W}$ was determined to be the optimal lysis time to ensure consistently high extraction efficiency of Pseudoloma neurophilia spores with minimal loss of signal due to DNA shearing (Fig. 2).

Extraction comparison. In Trial $\mathrm{A}$, the sonication pretreatment, both unextracted (mean $\mathrm{C}_{\mathrm{q}}=26.9$ ) and followed by QIAGEN extraction (mean $\mathrm{C}_{\mathrm{q}}=27.7$ ), resulted in a lower mean crossing threshold than other methods (Fig. 3). The multiple comparison with bestprocedure analysis of Trial B showed chitinase pretreatment followed by QIAGEN extraction (CQ), QIAGEN extraction with no pretreatment (Q), sonication pretreatment with no further extraction (S) and sonication pretreatment followed by QIAGEN extraction (SQ) to be in the best group, with sonication alone (S) to have the lowest mean crossing threshold (mean $\mathrm{C}_{\mathrm{q}}=$ 28.74). Sonication followed by QIAGEN extraction (mean $\mathrm{C}_{\mathrm{q}}=29.37$ ) had the next lowest mean crossing threshold (Fig. 3).

Detection limit. The assay, using the PN10F/R primer set and probe PN, consistently detected $<1$ spore (0.2 spore) per reaction in PBS when the sample was sonicated. This was determined based on the original number of spores (10 spores $\left.100 \mu \mathrm{l}^{-1}\right), 2 \mu \mathrm{l}$ of which were tested by real-time PCR. Pseudoloma neurophilia ssu rDNA was detected when spawn water, eggs, or sperm samples were spiked with spores at from 4 to 5 spores per real-time PCR reaction in all trials and replicates within trials (Table 2) using sonication followed by QIAGEN extraction. Parasite ssu rDNA was detected in spawn water spiked with as low as 10 spores $\mathrm{l}^{-1}$ (0.1 spore per reaction) in one trial.

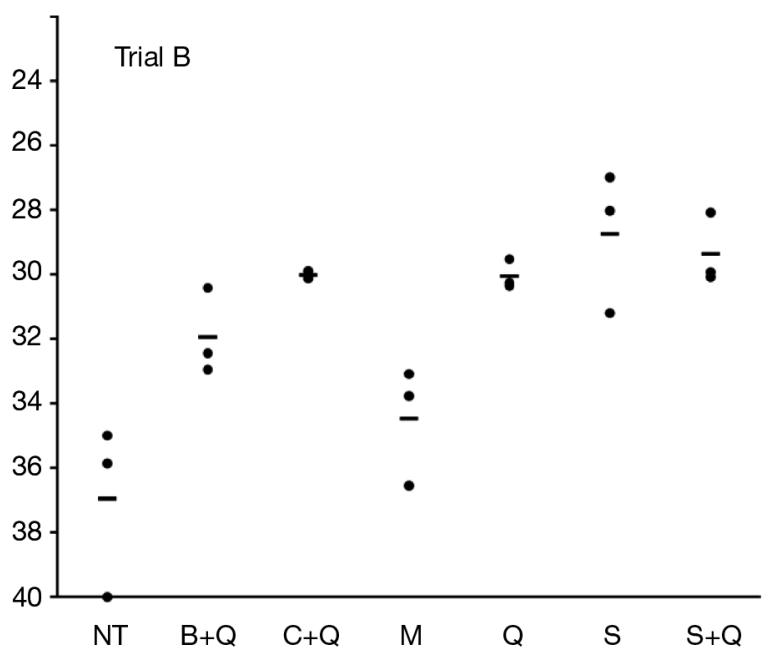

Fig. 3. Pseudoloma neurophilia. Comparison of spore extraction methods by quantification cycle threshold $\left(\mathrm{C}_{\mathrm{q}}\right)$. In Trial $\mathrm{A}$, the lowest mean $\mathrm{C}_{\mathrm{q}}(26.74)$ was shown by sonication treatment alone, followed by sonication with DNA extraction by QIAGEN silica gel membrane method (27.73). In Trial $B$, the lowest mean $C_{q}(28.74)$ was shown by sonication alone, followed by sonication and QIAGEN DNA extraction combined (29.93). NT: no treatment; M: MoBio; Q: QIAGEN; P: peroxide; S: sonication; B: beadbeating; $\mathrm{C}$ : chitinase. Bars: mean $\mathrm{C}_{\mathrm{q}}$ i points: individual sample $\mathrm{C}_{\mathrm{q}} \mathrm{s}$ 
Table 2. Pseudoloma neurophilia. Spiked sample results from 3 separate trials. Numbers for each trial represent quantification cycle threshold $\left(\mathrm{C}_{\mathrm{q}}\right)$ detected by real-time PCR per sample. Spawn water consisted of purified spores of $P$. neurophilia in $1 \mathrm{l}$ of water from a group spawn of specific pathogen-free (SPF) zebrafish. Eggs consisted of purified spores of $P$. neurophilia in $40 \mu \mathrm{l}$ aliquot (representing approximately 20 eggs based on an initial volume of 500 eggs $\mathrm{ml}^{-1}$ ) of homogenate made from eggs spawned by SPF zebrafish. Sperm samples consisted of purified spores of $P$. neurophilia in $5 \mu$ aliquot of sperm obtained by squeezing SPF zebrafish males. -: no copies of $P$. neurophilia small subunit ribosomal DNA (ssu rDNA) detected after 40 cycles

\begin{tabular}{|c|c|c|c|c|c|}
\hline Sample type & $\begin{array}{l}\text { Starting concentration } \\
\text { (spores per reaction) }\end{array}$ & Trial 1 & Trial 2 & Trial 3 & Mean \pm SD \\
\hline Spawn water & $\begin{array}{l}500(5) \\
100(1) \\
50(0.5) \\
10(0.1)\end{array}$ & $\begin{array}{c}36.7,35.0,36.4 \\
-,-, 39.9 \\
38.3,37.5,39.4 \\
-,-,-\end{array}$ & $\begin{array}{c}35.8,34.8,37.9 \\
39.4,37.4,38.8 \\
38.9,-,- \\
-,-,-\end{array}$ & $\begin{array}{c}35.6,36.1,37.9 \\
38.8,39.3,39.7 \\
37.3,-, 39.4 \\
38.1,38.5,-\end{array}$ & $\begin{array}{l}36.2 \pm 1.1 \\
39.0 \pm 0.8 \\
38.5 \pm 0.9 \\
38.3 \pm 0.3\end{array}$ \\
\hline Eggs & $\begin{array}{l}400(4) \\
40(0.4) \\
4(0.04)\end{array}$ & $\begin{array}{c}35.6,36.3,35.3 \\
38.6,-,- \\
-,-,-\end{array}$ & $\begin{array}{l}36.6,37.9,36.7 \\
36.2,36.8,36.0 \\
37.2,38.6,37.3\end{array}$ & $\begin{array}{c}36.1,36.5,36.2 \\
35.2,36.9,36.9 \\
36.6,37.6,-\end{array}$ & $\begin{array}{l}36.4 \pm 0.7 \\
36.7 \pm 1.1 \\
37.5 \pm 0.7\end{array}$ \\
\hline Sperm & $\begin{array}{l}500(5) \\
50(0.5) \\
5(0.05)\end{array}$ & $\begin{array}{c}32.7,32.3,31.9 \\
35.9,36.6,34.8 \\
36.4,-, 36.3\end{array}$ & $\begin{array}{l}35.5,-, 34.4 \\
38.0,-, 34.4 \\
36.8,-, 34.4\end{array}$ & $\begin{array}{c}32.8,32.1,31.3 \\
35.6,35.6,34.9 \\
35.1,-,-\end{array}$ & $\begin{array}{l}32.9 \pm 1.4 \\
35.7 \pm 1.2 \\
35.8 \pm 1.0\end{array}$ \\
\hline
\end{tabular}

(It was, however, not detected in the other two.) We detected the parasite in eggs spiked with 2 spores per egg ( 0.4 spores per reaction) in all trials and in all but one replicate. We also detected the parasite in spiked sperm samples, consistently detecting the parasite at 10 spores $\mu^{-1}$ of sperm (0.5 spore per reaction). The inconsistent detection at these lower levels of parasite is likely reflective of the sampling error inherent in dealing with such dilute concentrations.

Comparison to conventional assay. With the exception of one tissue sample, the sonication-real-time PCR approach detected Pseudoloma neurophilia ssu rDNA in at least $1 \log$ higher dilution than the conventional assay without sonication (Table 3). Analysis of all 6 samples by the real-time PCR method showed that sonication followed by DNA extraction using the QIAGEN DNeasy Blood and Tissue Extraction kit resulted in a

Table 3. Pseudoloma neurophilia. Comparison of P. neurophilia detection by new real-time PCR assay utilizing sonication pretreatment with conventional PCR assay described by Whipps \& Kent (2006). The real-time PCR assay described detected the parasite from 1 to 3 log dilutions higher than the conventional assay. +: P. neurophilia small subunit ribosomal DNA (ssu rDNA) detected; - : no $P$. neurophilia ssu rDNA detected

\begin{tabular}{|c|c|c|c|c|c|c|c|c|c|c|}
\hline \multirow[t]{2}{*}{ Ind. } & \multicolumn{5}{|c|}{ Conventional PCR } & \multicolumn{5}{|c|}{ Real-time PCR } \\
\hline & 0 & -1 & -2 & -3 & -4 & 0 & -1 & -2 & -3 & -4 \\
\hline 1 & + & - & - & - & - & + & + & + & - & - \\
\hline 2 & + & - & - & - & - & + & - & - & - & - \\
\hline 3 & + & + & - & - & - & + & + & + & + & - \\
\hline 4 & + & - & - & - & - & + & + & + & - & - \\
\hline 5 & + & - & - & - & - & + & + & - & - & - \\
\hline 6 & + & - & - & - & - & + & + & - & - & - \\
\hline
\end{tabular}

mean decrease of crossing threshold of 2.34, as compared to the same samples that were not sonicated.

Group spawn experiment. Pseudoloma neurophilia ssu rDNA was detected in the brain and spinal tissues of 6 of 10 adult fish in the group spawning experiment. Eggs were pooled and divided into 5 aliquots and $P$. neurophilia ssu rDNA was detected in one pool. In contrast, $P$. neurophilia ssu rDNA was detected in nine of ten 11 water samples from the spawning tank.

\section{DISCUSSION}

Our new real-time PCR test for Pseudoloma neurophilia, combined with pretreatment of samples by sonication, provides a sensitive test for evaluation of fish tissues and a non-lethal method for detecting spores in fish tissues, water, and spawning products. This is best illustrated by the decreased $\mathrm{C}_{\mathrm{q}}$ of samples using methods employing sonication (Fig. 3), indicating an increase in the number of ssu rDNA copies present. Comparing the test to the conventional PCRbased assay developed by Whipps \& Kent (2006), we found that it was from 10 to 100 times more sensitive, and our calculations indicated that it could detect $<1$ spore per reaction based on the number of spores spiked into PBS. This increased sensitivity was not necessarily due to conventional vs. real-time format or differences in primers, but more likely due to pretreatment by sonication, as discussed below.

Regarding specificity, we designed the primers so that they would be unique to Pseudoloma neurophilia. Also, we evaluated our test with Glugea anomala from the three-spined stickleback Gasterosteus aculeatus, and Pleistophora hyphessobryconis from the neon tetra 
Paracheirodon innesi, as these 2 Microsporidia might be found in fish research facilities. Three-spined sticklebacks are used in laboratory research, and we recently detected $P$. hyphessobryconis in 3 zebrafish colonies (Sanders et al. 2010). Additionally, a total of 278 fish from 6 separate populations housed in a zebrafish colony known to be free of $P$. neurophilia was tested using this real-time PCR-based assay, with histological analysis on individuals from the same populations performed in parallel (Kent et al. 2011). No fish from this population tested positive for $P$. neurophilia by real-time PCR or histology.

Whereas the purpose of the present study was to develop a real-time PCR-based assay, we found that the primer set used in this format also will work in a conventional PCR format as a positive/negative screening test. However, we did not determine the sensitivity of the primers using this conventional PCR format. Whereas quantification is not necessary for detection of the parasite, it may be pertinent to implement this feature for future studies on disease progression, transmission, and dose response, and for this reason the real-time PCR platform was developed. Furthermore, the use of the real-time PCR platform eliminates post-amplification handling of samples, decreasing the chance of cross-contamination by amplicons that might lead to false positive results, and reduces processing time for samples.

Current protocols for the detection of Pseudoloma neurophilia involve either direct observation of spores in wet-mount preparations or routine histological sections using either hematoxylin and eosin or special stains such as Fungi-Fluor (Kent \& Bishop-Stewart 2003), acid-fast (Ramsay et al. 2009), or Luna (Peterson et al. 2011). The development of a conventional PCRbased test (Whipps \& Kent 2006) has allowed researchers to detect lower levels of the parasite in fish tissues than is possible by traditional histological methods and has been used effectively to screen brood fish and progeny to establish an SPF zebrafish colony (Kent et al. 2009, Kent et al. 2011). These testing methods are relatively sensitive and specific, but were not evaluated in non-lethal formats. Many populations of zebrafish used in research consist of small numbers of difficult-to-breed mutant lines, and thus it is often impractical to lethally sample statistically significant numbers for diagnostic testing. For example, to screen a population of 100 fish, assuming a one or greater percent prevalence of infection in the population, 96 fish would need to be examined in order to reach $95 \%$ confidence in detecting the infection at a prevalence of $1 \%$ or greater (Simon \& Schill 1984, Kent et al. 2009). Therefore, detection of $P$. neurophilia in the fish's environment or in spawning products offers a practical and desirable alternative.
Several studies have been undertaken that incorporate various techniques for concentration and detection of microparasites in water. These include membrane filtration, continuous-flow centrifugation, and some in combination with immunomagnetic separation or PCR to detect microsporidia and other small parasites in large volumes of drinking or surface water (Bukhari et al. 1998, Fournier et al. 2000, Swales \& Wright 2000, Hallett \& Bartholomew 2006, Graczyk et al. 2007, Hoffman et al. 2007). Graczyk et al. (1997) described the use of cellulose-acetate membrane filtration of water followed by dissolution of the filter using acetone to concentrate and visualize Cryptosporidium oocysts. This approach for spore concentration provided a sensitive method for detecting Pseudoloma neurophilia in water.

Successful detection of microsporidia in environmental samples using PCR also requires efficient extraction of DNA from spores. A large portion of our study was therefore devoted to determination of the method that yields the highest amount of PCR product from spores. Both extraction comparison trials, each utilizing different sample handling, showed that sonication increased the overall sensitivity of the test. In the initial DNA extraction comparison, samples were pretreated in pools and aliquots were taken from these pools for purification in order to minimize the effects of sampling error inherent in dealing with such a small number of spores. We have found it occasionally difficult to obtain homogenous suspensions of Pseudoloma neurophilia spores, and this is likely attributable to some non-polar factors on the exterior of the spore wall, as we often find the spores clustered in wetmount preparations, particularly around the meniscus of air bubbles. The second DNA extraction comparison was based upon the results of the first trial and further confirmed that sonication resulted in much higher levels of extracted DNA, even taking into account potential sampling errors. Our results were consistent with those of Phelps \& Goodwin (2007), who showed that the amount of DNA obtained from another egg-associated fish microsporidium, Ovipleistophora ovariae, was increased over 500 times by sonicating spores as compared to Proteinase $\mathrm{K}$ digestion alone. As with their study, we observed numerous intact spores in preparations following the QIAGEN extraction, indicating that this method and others which depend solely on Proteinase $\mathrm{K}$ and detergent digestion is not effective for disrupting some microsporidian spores. This is further supported by the higher overall sensitivity obtained from tissue samples which had been sonicated prior to DNA extraction.

Although the extraction comparison showed that sonication without the additional step of DNA extraction using the QIAGEN kit had the highest sensitivity 
with purified spores, this step was necessary for extraction from tank water and eggs due to inhibitory elements found in these sample matrices as determined by spiking sonicated, unpurified samples (data not shown). While this inhibition could potentially be eliminated by diluting the samples or adding adjuncts such as bovine serum albumin, the DNA extraction step was added in order to maintain a consistent extraction, with samples potentially containing variable amounts of inhibitory substances and to avoid diluting the potential target.

Our test was also effective for spawning water, eggs, and sperm. We were able to detect very low levels of the parasite in replicates within trials and obtained similar results with independent trials. Zebrafish are spawned by placing pairs or groups into small tanks with screen bottoms overnight. The F1 progeny of surface-disinfected eggs is the main source for establishing new populations of zebrafish and introducing new lines into existing zebrafish colonies (Lawrence 2007), an approach that has been used for decades to avoid movement of salmonid pathogens (Stead \& Laird 2002, Kent \& Kieser 2003). For salmonids, spawning products are also screened for specific pathogens that are maternally transmitted (Miriam et al. 1997). The use of surface-disinfected eggs is likely to be effective for avoiding certain bacterial pathogens, but this practice has not stopped the spread of Pseudoloma neurophilia within zebrafish colonies. The reason for this is likely 2-fold: the ineffectiveness of levels of chlorine used for disinfection of eggs (Ferguson et al. 2007), and the maternal transmission route of the parasite. Other fish microsporidia are vertically transmitted (Phelps \& Goodwin 2008), and several lines of indirect and observational data indicate that there is a risk of maternal transmission of $P$. neurophilia, either to the egg directly or from the ovary to the water during spawning. These lines of evidence are: (1) observation of the parasite in several established facilities that use only F1 progeny from disinfected eggs, (2) the common appearance of the parasite in the ovaries and occasionally within eggs, and (3) the high susceptibility of larval zebrafish to $P$. neurophilia infection. We detected the parasite in water and eggs from the spawn tank of a population of brood fish with an infection prevalence of $60 \%$, providing further evidence of maternal transmission.

Given that resistant spores are abundant at spawning, the only way to reliably avoid maternal transmission is to identify infected broodfish or progeny with a highly sensitive diagnostic test. Screening of $10 \mathrm{~d}$ old fry and broodstock from several zebrafish lines has been used to establish a Pseudoloma neurophilia SPF facility at Oregon State University (Kent et al. 2009, 2011). Our test also has potential to be used for non- lethal screening of adult stocks. This non-lethal format is feasible, as adult zebrafish spawn prodigiously and paired spawning is often performed in $1 \mathrm{l}$ or less of water (Lawrence 2007, Harper \& Lawrence 2011). The results of the group spawn experiment illustrate the potential application of this assay in this format. However, it is important to note that testing spawning products and water may not be reliable for detecting the parasite in all infec ted fish. The parasite does not occur in the ovaries of all infected females (Kent \& BishopStewart 2003), and thus these fish may be less likely to shed spores at spawning. Also, we have not seen the parasite in the testis by histology, and thus this approach for non-lethal testing may not be useful for identifying infected males.

With these limitations, the test would still be of value in that a positive result from pooled samples from a group spawn would clearly demonstrate that at least one fish in the spawning group was positive. Moreover, our test is extremely sensitive, and thus a negative result with spawning water or eggs would be highly suggestive that the progeny from this particular spawn were not infected. Other experiments performed in our laboratory have produced similar results, and further studies are currently underway to elucidate the progression and transmission of the parasite within populations of fish. The purpose of this effort is to determine the predictive value of this type of test, and its reliability in detecting positive fish in lightly infected populations. We are also further investigating the role of males in maternal transmission of the parasite. Cryopreserved sperm is used for long-term storage of zebrafish lines and is frequently used to establish new populations (Westerfield 2007). Whereas we have not detected the parasite in the testis by histology, sperm could be contaminated during the squeezing process employed to obtain a sample. Hence, our new test provides a method for screening sperm for the parasite.

In conclusion, we provide here a new real-time PCRbased approach for Pseudoloma neurophilia that is more sensitive than previous tests. Additionally, we have developed a method by which to sample and detect the parasite in water, eggs, and sperm, thus providing the foundation for a non-lethal test. It is intended that personnel involved in the maintenance of laboratory Danio rerio populations use this protocol as a basis for their own testing protocols and modify it to suit the needs of individual facilities for monitoring and screening.

Acknowledgements. This study was supported by grants from the National Institutes of Health (NIH NCRR 5R24RR01738602 and NIH NCRR P40 RR12546-03S1). We thank C. Whipps, of the State University of New York, and T. Peterson, of Ore- 
gon State University, for manuscript review, C. Buchner, of the Sinnhuber Aquatic Research Laboratory, for providing negative control specimens, and G. Weaver, of the Oregon State University Department of Statistics, for statistical support.

\section{LITERATURE CITED}

Amatruda JF, Shepard JL, Stern HM, Zon LI (2002) Zebrafish as a cancer model system. Cancer Cell 1:229-231

Baker DG (1998) Natural pathogens of laboratory mice, rats, and rabbits and their effects on research. Clin Microbiol Rev 11:231-266

Brown AMV, Kent ML (2002) Molecular diagnostics for Loma salmonae and Nucleospora salmonis (Microsporidia). In: Cunningham C (ed) Molecular diagnosis of salmonid diseases. Kluwer Academic Publishers, London, p 267-283

Bukhari Z, McCuin RM, Fricker CR, Clancy JL (1998) Immunomagnetic separation of Cryptosporidium parvum from source water samples of various turbidities. Appl Environ Microbiol 64:4495-4499

Cornman RS, Chen YP, Schatz MC, Street C and others (2009) Genomic analyses of the microsporidian Nosema ceranae, an emergent pathogen of honey bees. PLoS Pathog 5: e1000466

> Delarbre S, Gatti S, Scaglia M, Dancourt M (2001) Genetic diversity in the microsporidian Encephalitozoon hellem demonstrated by pulsed-field gel electrophoresis. J Eukaryot Microbiol 48:471-474

> Docker MF, Devlin RH, Richard J, Khattra J, Kent ML (1997) Sensitive and specific polymerase chain reaction assay for detection of Loma salmonae (Microsporea). Dis Aquat Org 29:41-48

> Dooley K, Zon LI (2000) Zebrafish: a model system for the study of human disease. Curr Opin Genet Dev 10:252-256

> Dowd SE, Gerba CP, Pepper IL (1998) Confirmation of the human-pathogenic microsporidia Enterocytozoon bieneusi, Encephalitozoon intestinalis, and Vittaforma corneae in water. Appl Environ Microbiol 64:3332-3335

Eisen JS (1996) Zebrafish make a big splash. Cell 87:969-977

Ferguson JA, Watral V, Schwindt AR, Kent ML (2007) Spores of two fish microsporidia (Pseudoloma neurophilia and Glugea anomala) are highly resistant to chlorine. Dis Aquat Org 76:205-214

Fournier S, Liguory O, Santillana-Hayat M, Guillot E and others (2000) Detection of microsporidia in surface water: a one-year follow-up study. FEMS Immunol Med Microbiol 29:95-100

Graczyk TK, Cranfield MR, Fayer R (1997) Recovery of waterborne oocysts of Cryptosporidium from water samples by the membrane-filter dissolution method. Parasitol Res 83: $121-125$

> Graczyk TK, Sunderland D, Tamang L, Shields TM, Lucy FE, Breysse PN (2007) Quantitative evaluation of the impact of bather density on levels of human-virulent microsporidian spores in recreational water. Appl Environ Microbiol 73: 4095-4099

Hallett SL, Bartholomew JL (2006) Application of a real-time PCR assay to detect and quantify the myxozoan parasite Ceratomyxa shasta in river water samples. Dis Aquat Org 71:109-118

Harper C, Lawrence M (2011) The laboratory zebrafish. CRC Press, Boca Raton, FL

> Higes M, Martin R, Meana A (2006) Nosema ceranae, a new microsporidian parasite in honeybees in Europe. J Inver- tebr Pathol 92:93-95

Hill AJ, Teraoka H, Heideman W, Peterson RE (2005) Zebrafish as a model vertebrate for investigating chemical toxicity. Toxicol Sci 86:6-19

Hoffman RM, Wolk DM, Spencer SK, Borchardt MA (2007) Development of a method for the detection of waterborne microsporidia. J Microbiol Methods 70:312-318

Joseph J, Sharma S, Murthy SI, Krishna PV and others (2006) Microsporidial keratitis in India: 16S rRNA gene-based PCR assay for diagnosis and species identification of microsporidia in clinical samples. Invest Ophthalmol Vis Sci 47:4468-4473

Kent ML, Bishop-Stewart JK (2003) Transmission and tissue distribution of Pseudoloma neurophilia (Microsporidia) of zebrafish, Danio rerio (Hamilton). J Fish Dis 26:423-426

Kent ML, Kieser D (2003) Avoiding the introduction of exotic pathogens with Atlantic salmon Salmo salar reared in British Columbia. In: Lee CS, O'Bryen PJ (eds) Biosecurity in aquaculture production systems: exclusion of pathogens and other undesirables. World Aquaculture Society, Baton Rouge, LA, p 43-50

Kent ML, Whipps CM, Matthews JL, Florio D and others (2004) Mycobacteriosis in zebrafish (Danio rerio) research facilities. Comp Biochem Physiol Part C: Toxicol Pharmacol 138:383-390

Kent ML, Spitsbergen JM, Matthews JM, Fournie JW, Westerfield M (2007) Diseases of zebrafish in research facilities. Zebrafish International Resource Center, Eugene, OR, available at http://zebrafish.org/zirc/health/diseaseManual.php

Kent ML, Feist SW, Harper C, Hoogstraten-Miller S and others (2009) Recommendations for control of pathogens and infectious diseases in fish research facilities. Comp Biochem Physiol Part C: Toxicol Pharmacol 149:240-248

Kent ML, Buchner C, Watral V, Sanders JL, LaDu J, Peterson TS, Tanguay R (2011) Development and maintenance of a specific pathogen-free (SPF) zebrafish research facility for Pseudoloma neurophilia. Dis Aquat Org 95:73-79

Keohane EM, Weiss LM (1999) The structure, function, and composition of the microsporidian polar tube. In: Wittner M, Weiss LM (eds) The microsporidia and microsporidiosis. American Society for Microbiology, Washington, DC, p 196-224

Kuehl RO (2000) Design of experiments: statistical principles of research design and analysis, 2nd edn. Duxbury Press, Pacific Grove, CA, p 98-103

- Lawrence C (2007) The husbandry of zebrafish (Danio rerio): a review. Aquaculture 269:1-20

> Lindstrom NM, Call DR, House ML, Moffitt CM, Cain KD (2009) A quantitative enzyme-linked immunosorbent assay and filtration-based fluorescent antibody test as potential tools to screen broodstock for infection with Flavobacterium psychrophilum. J Aquat Anim Health 21: 43-56

> López-Vázquez C, Dopazo CP, Olveira JG, Barja JL, Bandín I (2006) Development of a rapid, sensitive and non-lethal diagnostic assay for the detection of viral haemorrhagic septicaemia virus. J Virol Methods 133:167-174

Matthews JL (2004) Common diseases of laboratory zebrafish. Methods Cell Biol 77:617-643

Matthews JL, Brown AMV, Larison K, Bishop-Stewart JK, Rogers P, Kent ML (2001) Pseudoloma neurophilia n. g., n. sp., a new microsporidium from the central nervous system of the zebrafish (Danio rerio). J Eukaryot Microbiol 48: $227-233$

Miriam A, Griffiths SG, Lovely JE, Lynch WH (1997) PCR and probe-PCR assays to monitor broodstock Atlantic salmon (Salmo salar L.) ovarian fluid and kidney tissue for pres- 
ence of DNA of the fish pathogen Renibacterium salmoninarum. J Clin Microbiol 35:1322

Müller A, Stellermann K, Hartmann P, Schrappe M and others (1999) A powerful DNA extraction method and PCR for detection of microsporidia in clinical stool specimens. Clin Diagn Lab Immunol 6:243-246

Peterson TS, Spitsbergen JM, Feist SW, Kent ML (2011) Luna stain, an improved selective stain for detection of microsporidian spores in histologic sections. Dis Aquat Org 95:175-180

Phelps NBD, Goodwin AE (2007) Validation of a quantitative PCR diagnostic method for detection of the microsporidian Ovipleistophora ovariae in the cyprinid fish Notemigonus crysoleucas. Dis Aquat Org 76:215-221

Phelps NBD, Goodwin AE (2008) Vertical transmission of Ovipleistophora ovariae (microspora) within the eggs of the golden shiner. J Aquat Anim Health 20:45-53

Ramsay JM, Watral V, Schreck CB, Kent ML (2009) Pseudoloma neurophilia infections in zebrafish Danio rerio: effects of stress on survival, growth, and reproduction. Dis Aquat Org 88:69-84

Sanders JL, Lawrence C, Nichols DK, Brubaker JF, Peterson TS, Murray KN, Kent ML (2010) Pleistophora hyphessobryconis (Microsporidia) infecting zebrafish Danio rerio in research facilities. Dis Aquat Org 91:47-56

Shaw RW, Kent ML, Adamson ML (2000) Viability of Loma

Editorial responsibility: Catherine Collins, Aberdeen, UK salmonae (Microsporidia) under laboratory conditions. Parasitol Res 86:978-981

Simon RC, Schill WB (1984) Tables of sample size requirements for detection of fish infected by pathogens: three confidence levels for different infection prevalence and various population sizes. J Fish Dis 7:515-520

Stead SM, Laird LM (2002). Handbook of salmon farming. Springer-Praxis, Aberdeen

Swales C, Wright S (2000) Evaluation of a continuous flow centrifuge for recovery of Cryptosporidium oocysts from large volume water samples. Water Res 34:1962-1966

Westerfield M (2007) The zebrafish book: a guide for the laboratory use of zebrafish (Danio rerio), 5th edn. University of Oregon Press, Eugene, OR

Whipps CM, Kent ML (2006) Polymerase chain reaction detection of Pseudoloma neurophilia, a common microsporidian of zebrafish (Danio rerio) reared in research laboratories. J Am Assoc Lab Anim Sci 45:36-39

Williams BA, Lee RCH, Becnel JJ, Weiss LM, Fast NM, Keeling PJ (2008) Genome sequence surveys of Brachiola algerae and Edhazardia aedis reveal microsporidia with low gene densities. BMC Genomics 9:200

Zhu X, Wittner W, Tanowitz HB, Kotler D, Cali A, Weiss LM (1993) Small subunit rRNA sequence of Enterocytozoon bieneusi and its potential diagnostic role with use of the polymerase chain reaction. J Infect Dis 168:1570-1575

Submitted: September 22, 2010; Accepted: May 25, 2011 Proofs received from author(s): August 22, 2011 